\title{
Evaluación del costo de electrificación rural en Bolivia para alcanzar el ODS 7
}

\section{Evaluation of the rural electrification cost in Bolivia to achieve SDG 7}

Miguel Fernández Fuentes ${ }^{1}$ (D). Evelyn Cardozo $^{2}$. Jaime Zambrana Vargas ${ }^{3}$. Gabriela Peña ${ }^{4}$. Sergio Balderrama ${ }^{5}$. Claudia Sánchez ${ }^{6}$. Alejandro Soto ${ }^{7}$. Sylvain Quoilin ${ }^{8}$.

${ }^{1}$ Investigador ENERGETICA. Cochabamba. Bolivia. miguel@energetica.org.bo

${ }^{2}$ Investigador CIE - UMSS. Cochabamba. Bolivia. evelyncardozo.r@fcyt.umss.edu.bo

${ }^{3}$ Investigador INCISO - UMSS. Cochabamba. Bolivia. jaimezambranavargas@ gmail.com

${ }^{4}$ Investigador CIE - UMSS. Cochabamba. Bolivia. gabrie@kth.se

${ }^{5}$ Investigador CIE - UMSS. Cochabamba. Bolivia. slbalderrama@ doct.uliege.be

${ }^{6}$ Investigador CIE - UMSS. Cochabamba. Bolivia. sanchez.solis.clau@gmail.com

${ }^{7}$ Investigador CIE - UMSS. Cochabamba. Bolivia. asm19971997@ gmail.com

${ }^{8}$ Investigador KU Leuven. Belgica. sylvain.quoilin@kuleuven.be

\section{RESUMEN}

El presente estudio cuantifica el desafío que supone el logro del objetivo 7 del desarrollo sostenible: Acceso a energía asequible y no contaminante (SDG7) en Bolivia. La solución planteada por el proyecto estima el costo total de provisión de energía eléctrica en todas las poblaciones de Bolivia, considerando factores socioeconómicos relevantes al realizar la planificación de la cobertura total para el país. El proyecto tiene dos etapas: la primera consiste en la recolección de datos y análisis de la demanda, así como de la percepción/visión de las poblaciones con relación en el uso de la energía; la segunda se centra en el análisis de la información y en la generación de resultados. La recolección de datos de campo que contemplan la demanda energética y el comportamiento en el uso y consumo de la energía se realizó en las comunidades de Raqaypampa (Cochabamba) y El Sena (Pando). Las mismas complementaron 


\title{
JOURNAL BOLIVIANO DE CIENCIAS - Vol. 17 - Número 50
}

ISSN: $2075-8944$

Universidad Privada del Valle - Bolivia

https://doi.org/10.52428/20758944.v17i50.2

información obtenida con anterioridad de El Espino (Santa Cruz) y Toconao (Altiplano frontera con Chile).

El Instituto de Investigaciones en Ciencias Sociales (INCISO), ENERGÉTICA y el Centro de Investigaciones en Energía (CIE), con la información recabada y los datos de indicadores del SDSN, estructuraron la composición estándar de una comunidad rural en Bolivia. Con esta información, el programa de generación de curvas de demanda RAMP simuló las demandas para poblaciones en las zonas bajas y altas de Bolivia, contrastando luego con diferentes tecnologías de electrificación; así se calculó el costo más eficiente de las soluciones encontradas. Finalmente, el costo para electrificar de manera total la población boliviana fue estimado y, en particular para electrificar al 100\% de la población aislada y dispersa que alcanza a 587 millones de dólares, dando cobertura a 273286 familias rurales.

Palabras clave: Acceso a la energía. Electrificación rural. Planificación energética. ODS 7. Sistemas fotovoltaicos domésticos.

\begin{abstract}
This study quantifies the challenge of achieving Sustainable Development Goal 7: Access to Affordable and Clean Energy (SDG7) in Bolivia. The solution proposed by the project finds the total cost of supplying electricity in all the towns of Bolivia, considering relevant socioeconomic factors when planning the total coverage for the country. The project has two stages: the first consists of data collection and analysis of the demand and the populations' perception/vision about the use of energy; the second focuses on the analysis of the information and the results generation. The collection of field data which includes energy demand and behavior in the use and consumption of energy was carried out in the communities of Raqaypampa (Cochabamba) and El Sena (Pando). They complemented information previously obtained from El Espino (Santa Cruz) and Toconao (Altiplano bordering Chile).
\end{abstract}

The Social Sciences Research Institute (INCISO), ENERGÉTICA and the Energy Research Center (CIE), with the information collected and the data on indicators from the SDSN, structured rural 


\section{JOURNAL BOLIVIANO DE CIENCIAS - Vol. 17 - Número 50}

ISSN: $2075-8944$

Universidad Privada del Valle - Bolivia

https://doi.org/10.52428/20758944.v17i50.2

communities' standard composition in Bolivia. With this information, RAMP, a demand profiles generation software, simulated the demands for populations in the low and high Bolivia areas, contrasting with different electrification technologies; thus, the most efficient solutions calculate. Finally, the cost to fully electrify the Bolivian population was estimated for electrify $100 \%$ of the isolated and dispersed population, which amounts to 587 million dollars, covering 273,286 rural families.

Keywords: Energy access. Rural electrification. Energy planning. SDG 7. Solar home systems.

\section{INTRODUCCIÓN}

Los 17 Objetivos del Desarrollo Sostenible, acordados por la Conferencia de Naciones Unidas en la reunión de Río de 2012, marcan los compromisos internacionales para erradicar la pobreza y mejorar la calidad de vida en el planeta (UNDP, 2018); entre ellos, el objetivo de desarrollo sostenible número siete (ODS 7) determina que para el año 2030 todos los habitantes del planeta deberían tener acceso a energía limpia y segura. En ese contexto, el Acceso Universal a la Energía y sus implicaciones con el resto de los ODS, hace que su cumplimiento sea estratégico para generar sinergias y potenciar otros logros, sociales, económicos, de salud, educación y ambientales.

En América Latina y el Caribe (ALAC) a 2018 la cobertura eléctrica alcanzó el 98,3\%; sin embargo, el pequeño porcentaje que aún falta por electrificar representa a una población de al menos 14 millones de personas ${ }^{1}$, las cuales están situadas mayoritariamente en zonas rurales y aisladas y tienen limitaciones a todos los servicios a los que se puede acceder gracias a la disponibilidad de una fuente moderna de energía como es la electricidad (CEPAL, 2017).

Los beneficios del acceso a la energía en área rurales en relación con mejorar los niveles de calidad de vida han sido ampliamente documentados en la región, tanto para proyectos de electrificación

1 Aunque el porcentaje de cobertura total tiene similitudes entre las diferentes fuentes, hay discrepancias sobre la cantidad total de personas que significa (CEPAL, 2017) (Vieira de Carvalho, 2015), existiendo diferencias de hasta 7 millones de personas. 
rural con redes (Jimenez, 2017), como aquellos que promueven energías renovables de aplicación descentralizada en diferentes países como Argentina (Scmukler y Garrido, 2016), Bolivia (Fernandez, 2010), o Perú (Arraiz y Calero, 2015), entre otros. Al año 2019, salvo Haití (38,7\%) y Honduras $(81,1 \%)$, el resto de los países está con coberturas entre 92,4\% y 99,9\%. Un aspecto común a todos los países (excepto Haití), es que la población sin acceso a electricidad es en la práctica población rural dispersa.

Por otro lado, la dificultad de lograr el acceso universal es manifiesta; en la medida que va creciendo la cobertura total, cada vez es más difícil lograr el acceso universal (Vieira de Carvalho, 2015); la Figura 1 muestra el crecimiento de la cobertura en ALAC entre 1998 y 2018, y permite apreciar tendencialmente, que la tasa anual de electrificación va disminuyendo.

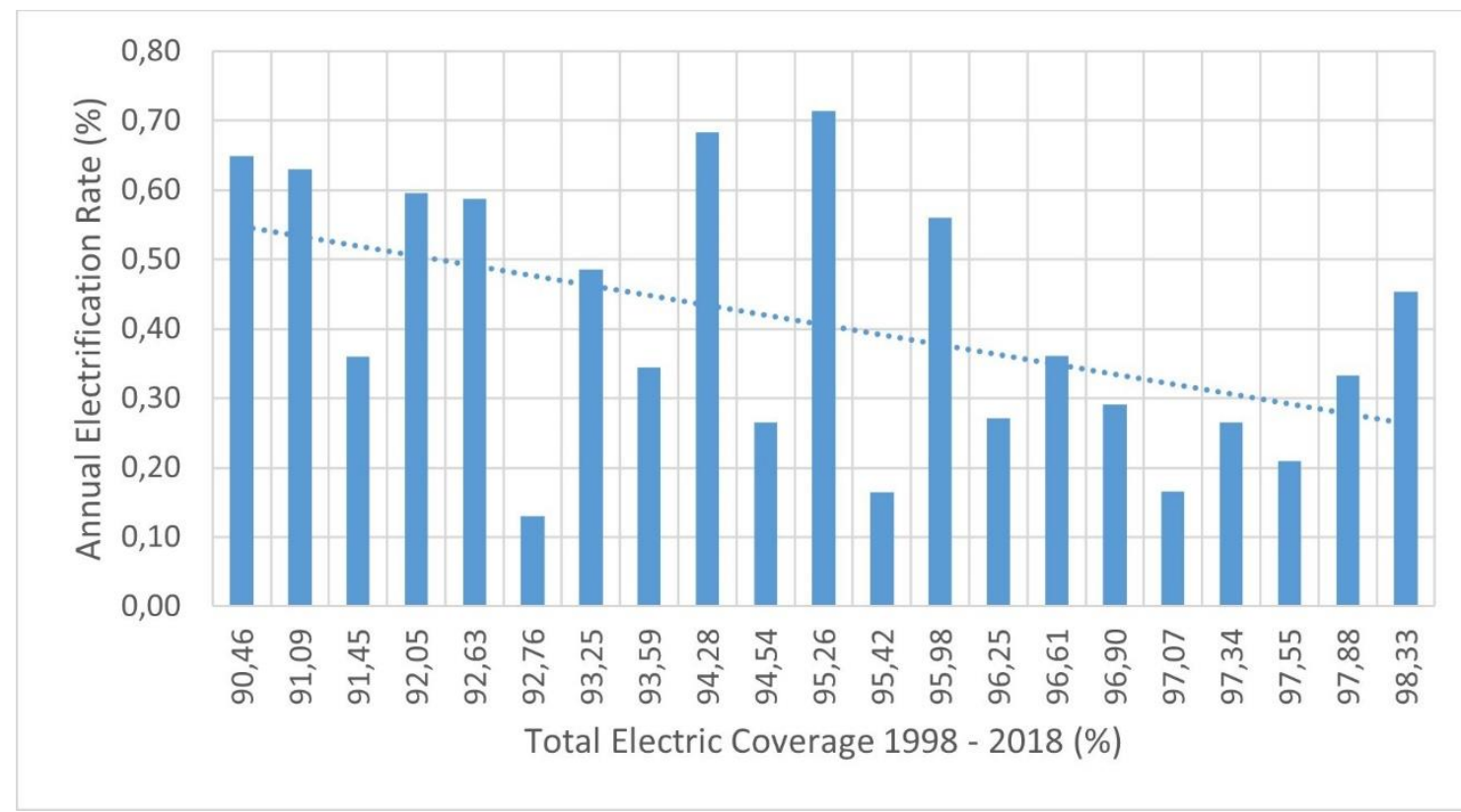

Figura 1. Tasa de electrificación anual Vs. Cobertura eléctrica en ALAC 1998 -2018

Fuente: Elaboración propia con base a datos del Banco Mundial, 202).

Bolivia cuenta con una geografía única con zonas climáticas contrastantes, sobre una superficie de $1098581 \mathrm{~km}^{2}$. El territorio se divide en tierras altas habitadas (hasta 4500 m.s.n.m.) y tierras bajas 


\section{JOURNAL BOLIVIANO DE CIENCIAS - Vol. 17 - Número 50}

ISSN: $2075-8944$

Universidad Privada del Valle - Bolivia

https://doi.org/10.52428/20758944.v17i50.2

(<800 m.s.n.m.), por su clasificación altitudinal. La población actual es de 11 millones de habitantes aproximadamente, de los cuales el 67,3\% vive en zonas urbanas y el 32,7\% en zonas rurales (Instituto Nacional de Estadística, 2019). La taza de electrificación aumentó en las últimas décadas, del 55\% en 1990 a cerca del 93\% en 2018 (Banco Mundial, 2020).

El gobierno nacional ha establecido una meta para alcanzar el 100\% de la tasa de electrificación para 2025, en línea con el ODS 7, para lo cual se requiere una estrategia nacional para guiar las necesidades de inversión. La Figura 2 muestra los conjuntos de datos de población, tasa de electrificación y líneas de transmisión en Bolivia. Las minirredes existentes en áreas densamente pobladas se ilustran en líneas moradas, mientras que las redes de distribución más pequeñas de microrredes no son visibles a esta escala.

Se hace una distinción entre minirredes y microrredes debido a que estas pueden variar en gran medida en tamaño (desde kilovatios hasta megavatios). En este estudio, las microrredes se refieren a sistemas más pequeños que las minirredes con pequeños sistemas de distribución no regulados. En Bolivia, las minirredes tienen un tamaño del orden de megavatios con grandes redes de distribución reguladas. Actualmente, la capacidad instalada de estos sistemas es de 141 MW, representando $6,8 \%$ de la capacidad instalada total del país, compuesta por una combinación energética de $66 \%$ de gas, $25 \%$ de diésel, $6 \%$ de energía hidroeléctrica y $4 \%$ de energía solar (Autoridad de Fiscalización y control Social de Electricidad, 2015). En los últimos años, se han incorporado varios sistemas de minirred a la red nacional, provocando una reducción en la huella de carbono (Ministerio de Hidrocarburos y Energía, 2014).

En este contexto, el objetivo de este trabajo es el de calcular el costo de llegar a un 100\% de cobertura eléctrica en Bolivia, utilizando las tecnologías más apropiadas y eficientes para una realidad social, económica propia de las áreas rurales dispersas de Bolivia. 
a.

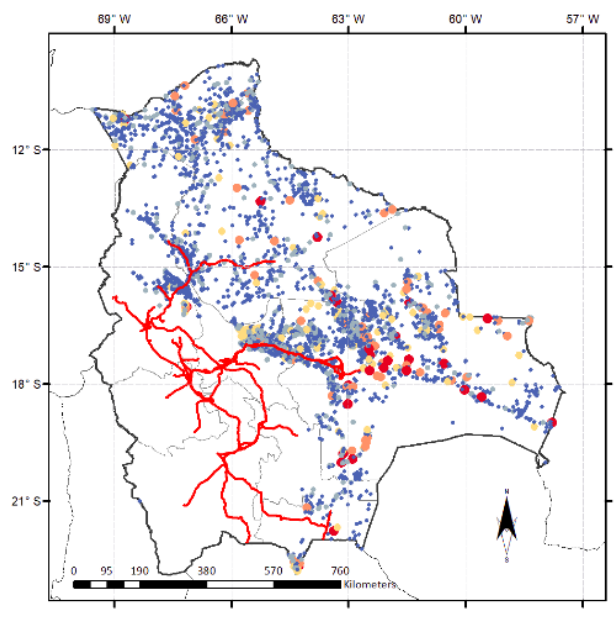

b.

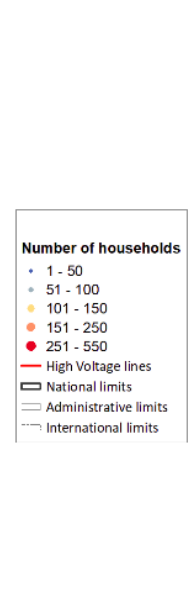

\section{.}

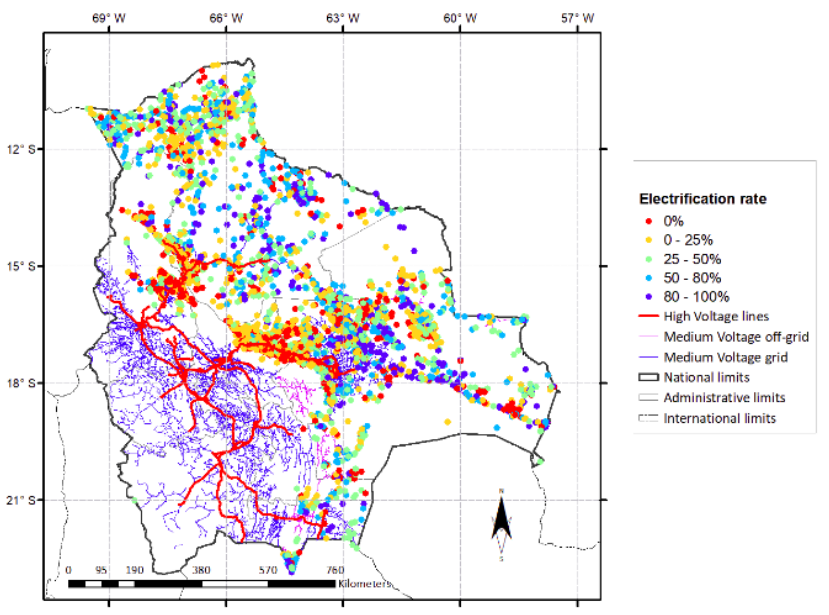

Figura 2. Conjunto de datos geoespaciales de comunidades, tasa de electrificación y red eléctrica existente en Bolivia: a) Tamaño de la población en cada comunidad y líneas de transmisión en 2017. Población extrapolada del Censo Nacional 2012, y b) Tasa de electrificación y líneas de transmisión de alto voltaje en 2012. Principales expansiones en transmisiones de AT entre los nodos oeste y centro

Fuente: Peña et al., 2020.

\section{METODOLOGÍA}

La complejidad geográfica de Bolivia y las diferentes necesidades de la población, implican la selección de alternativas de electrificación con base en los siguientes hechos: a) La densificación o expansión de la red principal es el método más recomendado, teniendo en cuenta que este tenga un costo adecuado; b) en lugares donde la red no puede llegar y que tienen una alta densidad poblacional las microrredes ofrecen una alternativa atractiva para poder satisfacer la demanda energética; c) cuando la densidad poblacional es muy baja, la alternativa más viable es el uso de sistemas de paneles fotovoltaicos domiciliarios para satisfacer las demandas básicas de electricidad (iluminación, acceso a radio y televisores, comunicación con telefonía celular), lo que tiene un efecto positivo sobre la calidad de vida de las personas. 


\section{JOURNAL BOLIVIANO DE CIENCIAS - Vol. 17 - Número 50 \\ ISSN: 2075-8944 \\ Universidad Privada del Valle - Bolivia \\ https://doi.org/10.52428/20758944.v17i50.2}

Lograr un $100 \%$ de cobertura a nivel nacional es una tarea compleja, ya que se necesita información específica para realizar la planificación. En este trabajo se utiliza en la metodología que se muestra en la Figura 3.

De manera resumida, el trabajo se enfoca en encontrar información que permita caracterizar las poblaciones típicas de Bolivia, para luego generar escenarios de demandas, disponibilidad de energía solar y otras características que permitan utilizar modelos simplificados. Finalmente, se usó OnSSET para calcular el costo de lograr 100\% de la cobertura eléctrica en Bolivia.

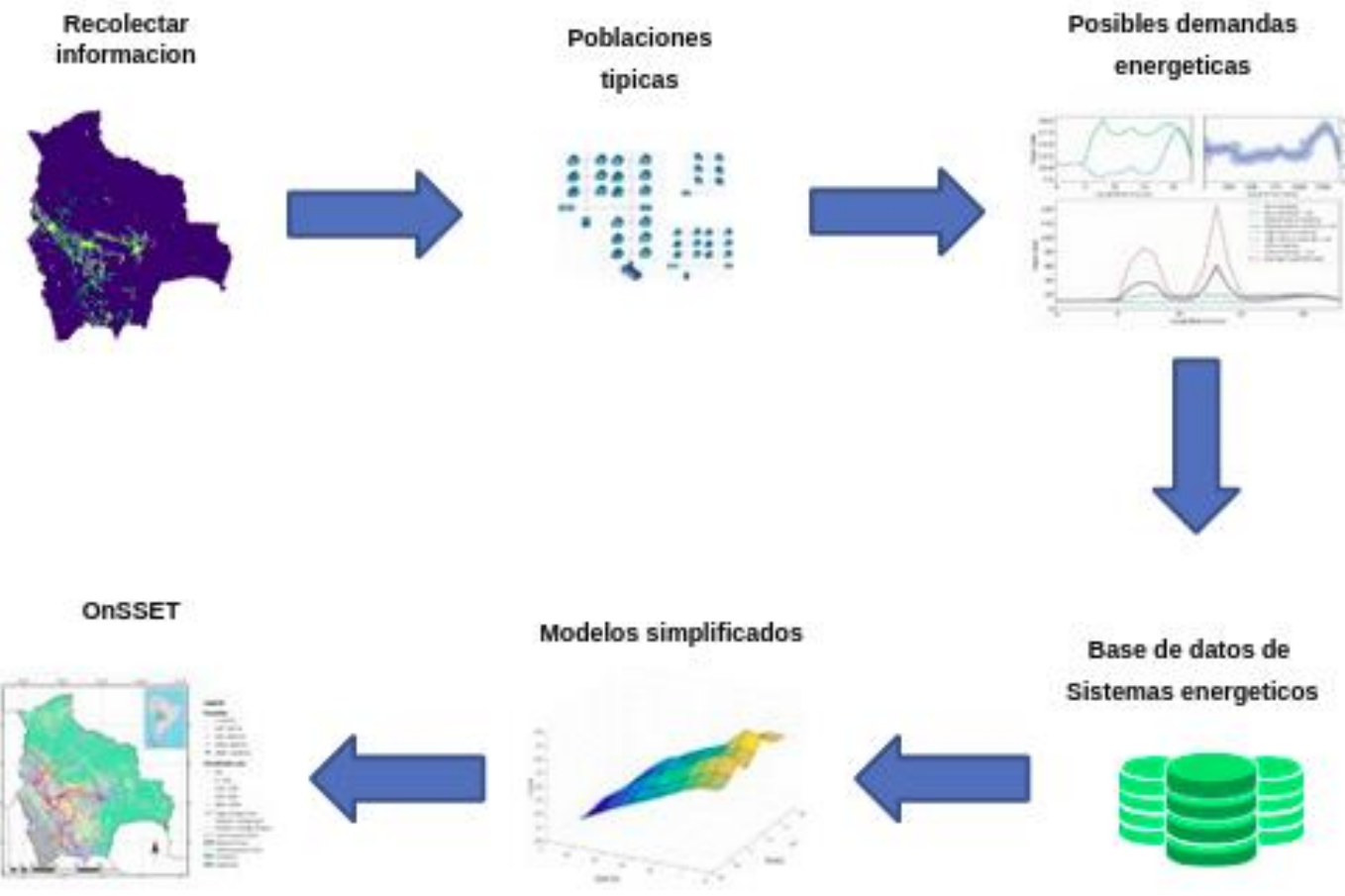

Figura 3. Diagrama de flujo de la metodología propuesta

Fuente: Elaboración propia con base a Peña et al., 2020.

\subsection{Recolección de información}

Los siguientes datos fueron extraídos del Censo Nacional de Bolivia de 2012 (datos georreferenciados) y del Atlas Municipal de los ODS en Bolivia de la SDSN:

- Número de hogares.

- Estado de electrificación. 


\section{JOURNAL BOLIVIANO DE CIENCIAS - Vol. 17 - Número 50}

ISSN: $2075-8944$

Universidad Privada del Valle - Bolivia

https://doi.org/10.52428/20758944.v17i50.2

- Fuente de electricidad (red, mini red, panel fotovoltaico, generador diésel).

- Ubicación geográfica de 19280 comunidades (Cobertura del Servicio Eléctrico a Nivel Comunidades Censo 2012, n.d.).

Se diferenció, además, entre zonas rurales y urbanas. Se aclara que la mayoría de las zonas con personas sin electricidad se concentran en las zonas rurales, por lo que se recabó información sobre la demanda energética en estas zonas, primeramente, se utiliza encuestas realizadas a 2 comunidades previas al estudio: Toconao (Altiplano, frontera con Chile) y El Espino (Chaco, en Santa Cruz). Adicionalmente, se realizaron 2 encuestas a las poblaciones de Raqaypampa (Valle de Mizque en Cochabamba) y El Sena (Amazonia en Pando). Con la realización de estas encuestas se buscaba tener una noción sobre los hábitos de consumo eléctrico, los equipos eléctricos que usa cada familia y algunas percepciones sociales sobre cuánta energía eléctrica requieren.

La información de la demanda de electricidad en zonas urbanas estuvo basada en estadísticas nacionales (Autoridad de Fiscalización y control Social de Electricidad, 2015). Por otro lado, para complementar la demanda en cada comunidad, se recopiló la demanda de electricidad de las instituciones comunitarias (centros de educación y salud) y de los servicios públicos (alumbrado público).

\subsection{Poblaciones típicas}

Las poblaciones de áreas rurales fueron caracterizadas de acuerdo con la información socioeconómica derivada de los mapas de pobreza (Catálogo GeoBolivia - GeoBolivia, n.d.) que divide en 5 categorías a Bolivia, basándose en el método multidimensional de necesidades básicas insatisfechas (Feres y Mancero, n.d.).

La población por encima del umbral de pobreza se clasificó como la categoría de ingresos altos (IA) y la población restante como la categoría de ingresos medios (IM). Por otro lado, se determinaron las comunidades que no tienen acceso a electricidad o que están parcialmente electrificadas de acuerdo con información del censo del 2012. Se distinguió entre comunidades densamente pobladas ( $>1000$ hogares) y comunidades de baja población (<1000 hogares). 


\section{JOURNAL BOLIVIANO DE CIENCIAS - Vol. 17 - Número 50}

ISSN: $2075-8944$

Universidad Privada del Valle - Bolivia

https://doi.org/10.52428/20758944.v17i50.2

Solamente a las comunidades de baja población se las dividió en tierras altas (800 - 4000 m.s.n.m.) y las tierras bajas (<800 m.s.n.m.). Estas comunidades de tierras bajas fueron además divididas entre la amazonia (Latitud $>-18^{\circ}$ ) y el chaco (Latitud $<-18^{\circ}$ ).

\subsection{Posibles demandas energéticas}

Estimar la demanda energética de poblaciones que probablemente hayan tenido un contacto limitado a equipos que funciona con electricidad es un desafío qué junto a la poca información disponible en Bolivia sobre este tema, hace de esta tarea un reto importante. Para resolver este problema, se ha revisado información y, en la literatura se registra la aplicación de modelos de generación de curvas de demanda basado en encuestas, con éxito (Mandelli et al., 2016).

Para este trabajo, se utiliza un modelo de generación de curvas de carga (RAMP) ideado para la estimación de la demanda energética en comunidades rurales (Lombardi et al., 2019). La idea básica del modelo es la simulación de los aparatos que usan electricidad en una casa u otro tipo de entidades existentes en una comunidad. Para esto, toma la potencia nominal de un electrodoméstico, periodos de uso, ciclos de trabajo entre otros. Finalmente, genera las curvas de demanda recreando el uso de los equipos durante el día. Un mayor detalle de cómo funciona el modelo y algunos ejemplos se encuentra en Lombardi et al. (2019).

\subsection{Generación de base de datos de sistemas optimizados}

Para poder generar modelos simplificados es necesario contar con una base de datos con las variables dependientes a estimar y sus correspondientes variables independientes. Para esto, se hizo un análisis previo en el cual se concluyó que solo 14 comunidades sin acceso a la electricidad tienen más de 560 hogares. Adicionalmente, es muy probable que comunidades con menos de 40 hogares se encuentren dispersas y sea muy costoso la instalación de redes de distribución.

Por lo tanto, el análisis se enfoca en comunidades entre 40 a 560 hogares. Para generar esta base de datos se aplicó la metodología desarrollada en Balderrama et al. (2020), que consiste en crear una serie de escenarios posibles y usar un modelo de dimensionamiento de sistemas aislados, de manera de poder encontrar la composición de la microrred que minimiza el costo para cada caso (Figura 4). 


\section{JOURNAL BOLIVIANO DE CIENCIAS - Vol. 17 - Número 50}

ISSN: $2075-8944$

Universidad Privada del Valle - Bolivia

https://doi.org/10.52428/20758944.v17i50.2

Se necesitan tres piezas de información para poder caracterizar un escenario: parámetros tecnoeconómicos, una curva de demanda y la disponibilidad de energía de los paneles fotovoltaicos. Los parámetros tecno-económicos que se utilizaron se encuentran expuestos en la Tabla 1. De manera de ser eficientes, se utilizó un Latin hyper-cube como técnica de muestreo (100 muestras). Para la demanda se tuvo como variable la cantidad de hogares en el rango de 50 a 550 con un paso de 50. Cuando se aplicó los modelos simplificados se extendió al rango encontrado antes (40 a 560 hogares). La disponibilidad solar se descargó de la página: https://www.renewables.ninja/. Se utilizaron coordenadas de poblaciones sin electricidad en cada optimización.

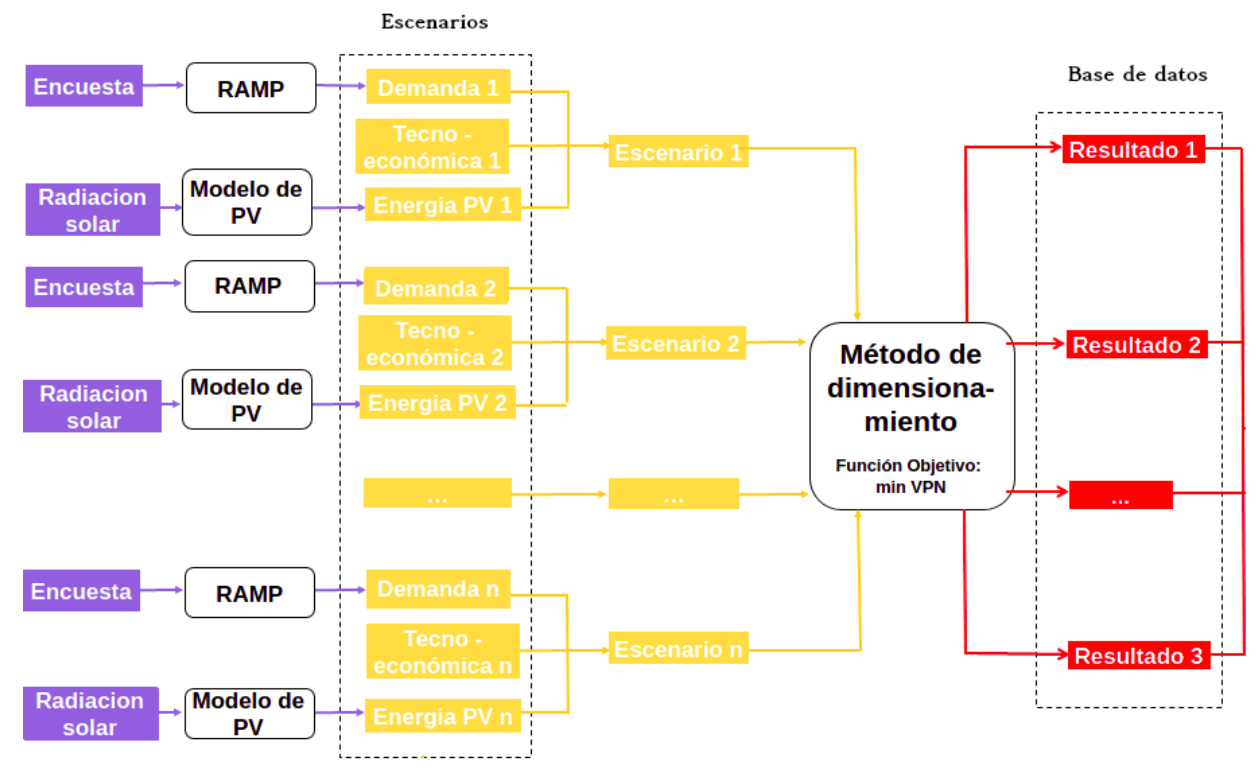

Figura 4. Metodología para la creación de escenarios

Fuente: Modificado de Balderrama et al., 2020.

\subsection{Modelos de regresión}

Una vez definido un escenario, es posible encontrar los componentes de una micro-red que minimice el costo total del sistema. Este proceso puede tomar unos pocos minutos a varias horas dependiendo de la técnica seleccionada. Si se toma en cuenta la cantidad de comunidades sin electricidad en Bolivia, aplicar esta técnica podría durar horas. Para evaluar cientos de comunidades en poco tiempo y sin perder la confiabilidad se usa una regresión gaussiana multivariable para crear modelos simplificados. Estos modelos son capaces de predecir diferentes 


\section{JOURNAL BOLIVIANO DE CIENCIAS - Vol. 17 - Número 50}

ISSN: $2075-8944$

Universidad Privada del Valle - Bolivia

https://doi.org/10.52428/20758944.v17i50.2

resultados de un proceso de dimensionamiento basados solo en los parámetros de entrada. Para este trabajo se crean modelos para calcular el costo neto presente de los sistemas aislados y la capacidad instalada de paneles fotovoltaicos. Para una mayor información se presenta en Balderrama et al. (2020).

\begin{tabular}{|c|c|c|}
\hline Parámetro (1) & Unidad & Rango \\
\hline $\begin{array}{c}\text { Costo unitario Panel Fotovoltaico (incluye cables, } \\
\text { estructuras y controles) }\end{array}$ & USD/kW & $1000-2000$ \\
\hline Costo unitario Batería de Litio FeP & USD/kWh & $400-600$ \\
\hline Costo unitario Generador de diésel & USD/kW & $1000-2000$ \\
\hline Costo de diésel & USD/l & $0,54-2$ \\
\hline
\end{tabular}

(1) Los rangos de precios máximos y mínimos corresponden a situaciones que se pueden encontrar en el mercado boliviano en función de: a) la cantidad, b) la distancia al centro de consumo c) la disponibilidad; de acuerdo con relevamiento realizado de campo en 2019.

Tabla 1. Parámetros mutables de costos para la creación de la base de datos

Fuente: Elaboración propia, 2021.

\subsection{Cálculo del costo de electrificación para Bolivia}

Una vez realizado el proceso de creación de los modelos simplificados, es posible usarlos en el software OnSSET para calcular el costo total de electrificación de Bolivia (Peña et al., 2020), utilizando información recolectada de diversas fuentes. Se destaca la información obtenida del censo 2012 como la población de las comunidades, estatus de electrificación además de su ubicación geográfica. También se cuenta con información tecno-económica de las diferentes tecnologías empleadas en este estudio.

En Bolivia, la extensión y densificación de la red ha sido el principal método para la electrificación de la población en el periodo 2012 al 2019, por esta razón se usará el algoritmo de extensión de red OnSSET (Mentis et al., 2017). Para esto se usan las líneas de media y alto voltaje construidas hasta la fecha y se procederá a electrificar a todas las poblaciones a una distancia menor a $50 \mathrm{~km}$ de estas líneas, hasta alcanzar el $92 \%$ de la población. 


\section{JOURNAL BOLIVIANO DE CIENCIAS - Vol. 17 - Número 50}

ISSN: $2075-8944$

Universidad Privada del Valle - Bolivia

https://doi.org/10.52428/20758944.v17i50.2

A continuación, se utilizan los modelos simplificados para estimar los costos de electrificar a las poblaciones entre 40 y 560 hogares con micro-redes. Finalmente, se dota al resto de comunidades con sistemas fotovoltaicos y baterías estimándose también la inversión necesaria. Con las tecnologías definidas, se procede a calcular las potencias instaladas y otros indicadores de interés.

\section{RESULTADOS}

Es importante resaltar que, debido a la gran cantidad de información procesada solo los resultados más relevantes son expuestos en este documento. El resto de la información y herramientas necesarias para reproducir esta investigación serán puestas a disposición del público al finalizar el proyecto en el siguiente repositorio: https://github.com/CIE-UMSS/SDSN_PROJECT_2019.

\subsection{Resultados de las encuestas}

Como se explicó anteriormente, se realizaron 2 encuestas a poblaciones rurales para poder entender las dinámicas que empujan el consumo eléctrico. La encuesta en Raqaypampa se realizó entre el 4 al 7 de octubre del 2019 y la encuesta en El Sena se realizó entre el 28 de septiembre al 7 de octubre del 2019. Durante el transcurso de estas encuestas se preguntó sobre la tenencia de equipos eléctricos, hábitos de las personas, expectativas de uso. Adicionalmente, en Rayqaypampa se levantó información sobre el consumo eléctrico de centros de servicios y productivos.

\subsubsection{Resultados de la encuesta en Raqaypampa}

El territorio de Raqaypampa tiene una extensión de $556 \mathrm{~km}^{2}$, está ubicado entre las coordenadas de longitud oeste $65^{\circ} 18^{\prime}$ a $65^{\circ} 32^{\prime}$ y latitud sud $18^{\circ} 04^{\prime}$ a $18^{\circ} 24^{\prime}$. Sus límites son: al norte con el municipio de Mizque, al sur con el departamento de Potosí, al este con el municipio de Aiquile y al oeste con el municipio de Mizque. La zona oscila de 1670 m.s.n.m. a 3448 m.s.n.m. y es parte de la región de los valles.

Después de un largo proceso de lucha de la población y de las autoridades de la organización sindical han logrado la constitución como Autonomía Indígena Originaria Campesina mediante la consolidación y reconocimiento como Territorio Indígena Originario Comunitario (TIOC) en el marco del desarrollo del Estado Plurinacional. 
Durante el trabajo de campo en Raqaypampa, se pudo realizar 50 diferentes encuestas a hogares. En la Tabla 2, se puede encontrar la información más relevante. En general una casa en Raqaypampa existen 4 individuos de diferentes rangos de edad. Cada una de estas personas impacta de manera diferente en el consumo eléctrico. Se puede esperar que un niño de 5 años o menos vea la televisión en cualquier momento del día y alguien de 17 solamente en las tardes. Así también, es posible un mayor consumo en familias que tienen niños/jóvenes en edad escolar durante el periodo de vacaciones.

\begin{tabular}{|c|c|c|}
\hline Información & Cantidad & Promedio \\
\hline Niños (0 a 5 años) & 23 & 0,5 \\
\hline $\begin{array}{c}\text { Niños/adolescentes (5- } \\
17 \text { años) }\end{array}$ & 73 & 1,5 \\
\hline Adultos & 87 & 1,74 \\
\hline Personas mayores & 15 & 0,3 \\
\hline Numero de cuartos & 144 & 2,9 \\
\hline Casas de adobe & 47 & 0,94 \\
\hline $\begin{array}{c}\text { Costo promedio de } \\
\text { electricidad (Bs/mes) }\end{array}$ & - & 30 \\
\hline
\end{tabular}

Tabla 2. Parámetros mutables de población de Raqaypampa para la creación de la base de datos

Fuente: Elaboración propia, 2021.

Es relevante reportar que 38 hogares contaban con radios y solamente 25 contaban con televisores. Existe una gran diferencia en el uso de televisores, ya que el rango de uso varía entre 1 a 10 horas en diferentes hogares. El extendido uso de los celulares es confirmado en Raqaypampa debido a que 48 hogares respondieron que tienen 1 o más celulares.

En cuanto a equipos de alto consumo, se reportan 15 refrigeradores, 2 bombas de agua, 3 molinos y algunos equipos de metalmecánica. En cuanto a hábitos de cocina, 6 hogares reportaron cocinar en una habitación abierta, 23 adentro o afuera dependiendo de la ocasión y el resto en una 
habitación cerrada. Casi todos los hogares usan una combinación de gas y leña. Finalmente, a pesar de que Raqaypampa es un lugar frío por su ubicación, ningún hogar utiliza algún artefacto para calentarse.

\subsubsection{Resultados de la encuesta en El Sena}

La comunidad de El Sena, se encuentra en la capital del municipio del mismo nombre, en la provincia Madre de Dios del Departamento de Pando; ubicada geográficamente en las coordenadas $11^{\circ} 29^{`} 34^{\prime}$ S de latitud sur, 67\%14 52” O de longitud oeste, el poblado se encuentra a una altura media de 174 m.s.n.m., con un clima típico del oriente boliviano, con precipitaciones, una humedad media a alta y fuerte radiación solar.

Durante el trabajo de campo en El Sena, se pudo realizar 30 diferentes encuestas a hogares. En la Tabla 3, se puede encontrar la información más relevante.

\begin{tabular}{|c|c|c|}
\hline Información & Cantidad & Promedio \\
\hline Niños (0 a 5 años) & 30 & 1 \\
\hline $\begin{array}{c}\text { Niños/adolescentes (5- } \\
17 \text { años) }\end{array}$ & 58 & 2 \\
\hline Adultos & 61 & 0,1 \\
\hline Personas mayores & 3 & 3 \\
\hline Numero de cuartos & 90 & 75 \\
\hline Costo promedio de & - & \\
\hline electricidad (Bs/mes) & & \\
\hline
\end{tabular}

Tabla 3. Parámetros mutables de población de El Sena en la creación de la base de datos

Fuente: Elaboración propia, 2021.

En general en un hogar en El Sena existe 5 individuos de diferentes rangos de edad. Existe una mayor cantidad de personas en el Sena que en Raqaypampa, también el dinero que invierten en electricidad es mayor. 
A diferencia de Raqaypampa, en El Sena se tiene solamente 4 radios y 33 televisores, los cuales son más usados que en Raqaypampa. 39 celulares existen en El Sena, 22 refrigeradores, 4 bombas de agua. En El Sena, solamente 1 entrevistado cocina en un cuarto abierto y todos menos 1 cocina con leña. El calor es sofocado con 19 ventiladores presentes en la comunidad. Al analizar toda la información en su conjunto se puede decir que El Sena es un lugar donde existe mayor demanda y más movimiento de dinero por la cantidad de equipos eléctricos presentes.

\subsubsection{Contrastes entre Raqaypampa y El Sena}

Más allá de las diferencias climatológicas y geográficas, una comparación entre ambas comunidades sobre aspectos poblacionales se aprecia en la Figura 5, donde se visualiza que El Sena es una población más joven y numerosa por hogar que Raqaypampa. Esto repercutirá en el consumo energético posteriormente.

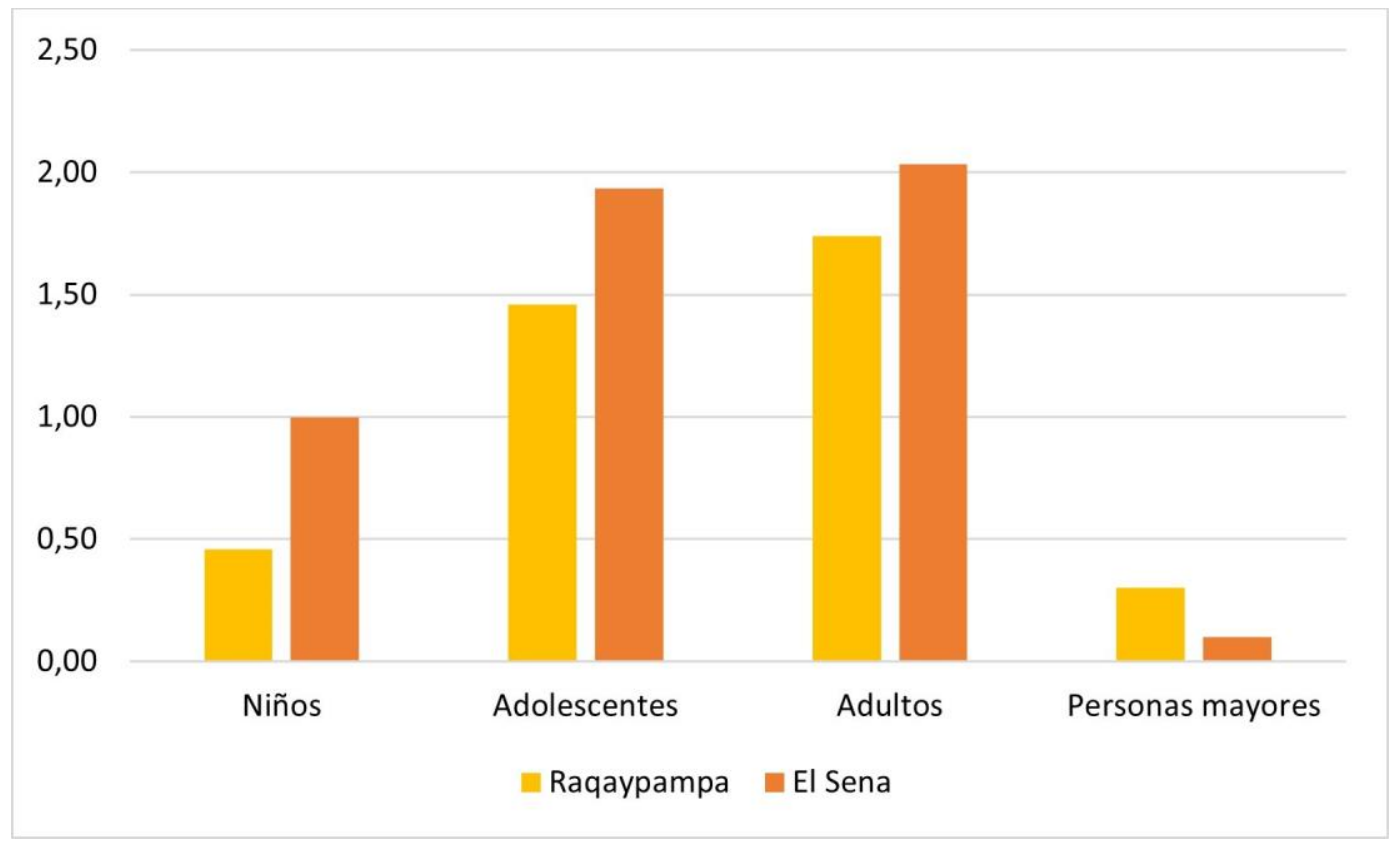

Figura 5. Composición promedio de un hogar

Fuente: Elaboración propia, 2021. 
La Figura 6 muestra la tenencia de equipos eléctricos por hogar en promedio, donde resalta también la diferencia entre ambas comunidades, y el mayor uso de equipos eléctricos en El Sena (más de un televisor por hogar, más de un celular por hogar, 73\% de los hogares usan refrigeradores y $63 \%$ usan ventiladores), esto sin duda condicionado por los aspectos climáticos propios de la región.

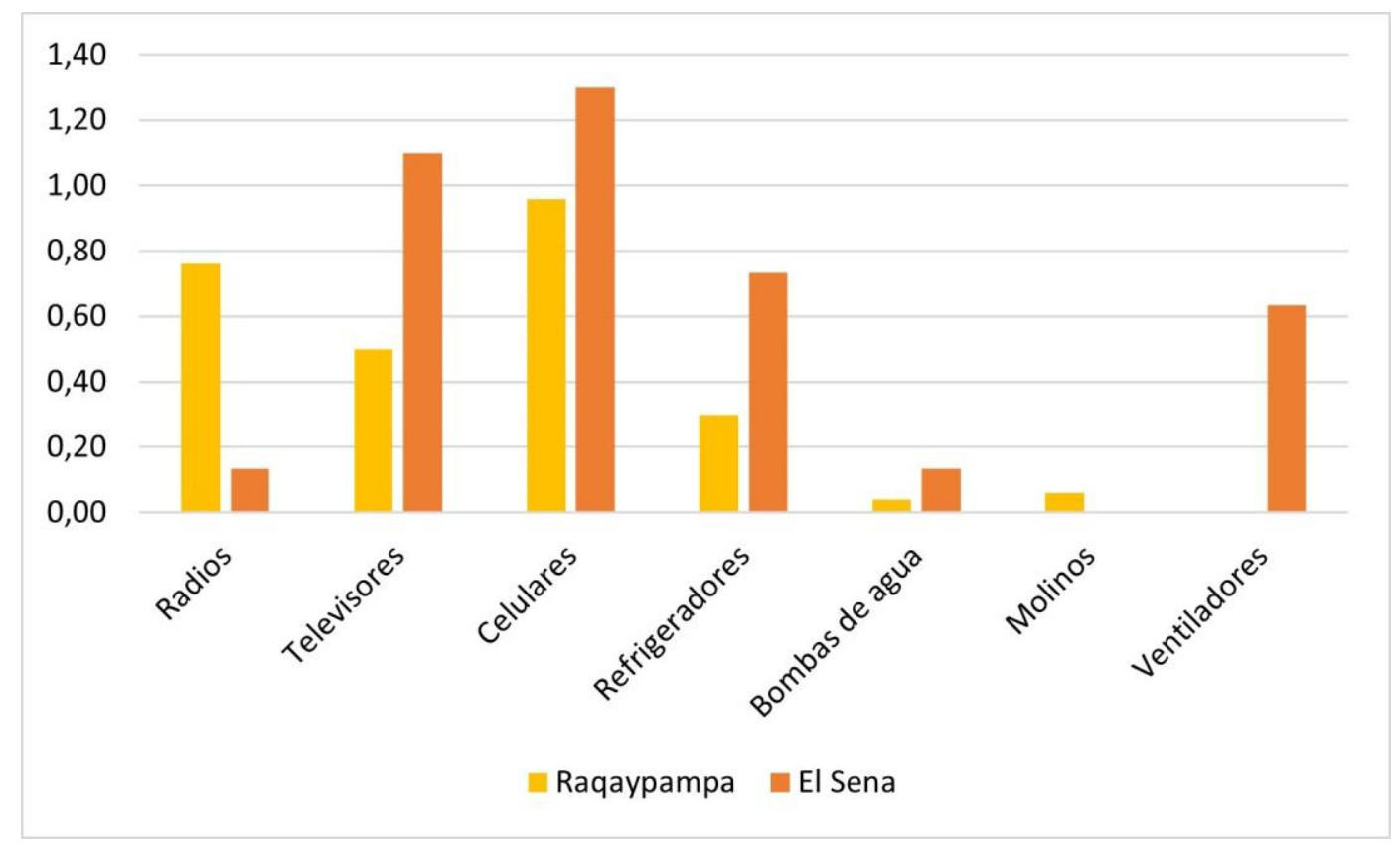

Figura 6. Equipos Eléctricos: tenencia promedio por hogar

Fuente: Elaboración propia, 2021.

\subsubsection{Comunidades típicas en Bolivia}

Con la información disponible es posible crear una población típica en Bolivia que está compuesta por personas con ingresos altos (IA) y medios (IM), un colegio, un centro de salud, iluminación pública y una iglesia. En la Tabla 4, se encuentra los artefactos típicos de cada una de las clases definidas de tres regiones de Bolivia (amazonas, tierras altas y chaco). La cantidad de equipos en cada uno de los hogares típicos representan la cantidad esperada de estos. Tomando en cuenta una cantidad que permita limitar la pobreza energética de los pobladores. En las encuestas y por la experiencia de los autores, se sabe que probablemente muchos hogares tendrán una menor cantidad de equipos, pero realizar una planificación sin tomar en cuenta la mínima cantidad de energía 
JOURNAL BOLIVIANO DE CIENCIAS - Vol. 17 - Número 50

ISSN: $2075-8944$

Universidad Privada del Valle - Bolivia

https://doi.org/10.52428/20758944.v17i50.2

necesaria para llevar una vida decente puede llevar a frustración y desconfianza de estas comunidades marginadas.

\begin{tabular}{|c|c|c|c|c|c|c|}
\hline Equipo & $\begin{array}{c}\text { IA } \\
\text { Tierras } \\
\text { altas }\end{array}$ & $\begin{array}{c}\text { IM } \\
\text { Tierras } \\
\text { altas }\end{array}$ & $\begin{array}{c}\text { IA } \\
\text { Chaco }\end{array}$ & $\begin{array}{c}\text { IM } \\
\text { Chaco }\end{array}$ & $\begin{array}{c}\text { IA } \\
\text { Amazonas }\end{array}$ & $\begin{array}{c}\text { IM } \\
\text { Amazonas }\end{array}$ \\
\hline Foco interno & 6 & 2 & 6 & 2 & 3 & 3 \\
\hline Foco externo & 2 & 1 & 2 & 1 & 2 & 2 \\
\hline TV & 2 & 1 & 2 & 1 & 1 & 1 \\
\hline Radio & 1 & 1 & 0 & 0 & 0 & 0 \\
\hline $\begin{array}{l}\text { Cargador de } \\
\text { celular }\end{array}$ & 4 & 1 & 0 & 1 & 2 & 2 \\
\hline Refrigerador & 1 & 0 & 0 & 0 & 1 & 1 \\
\hline Laptop & 1 & 0 & 1 & 0 & 0 & 0 \\
\hline Plancha & 1 & 1 & 0 & 0 & 0 & 0 \\
\hline DVD & 0 & 0 & 1 & 1 & 0 & 0 \\
\hline Antena & 0 & 0 & 1 & 1 & 0 & 0 \\
\hline Batidora & 0 & 0 & 1 & 0 & 1 & 1 \\
\hline Ventilador & 0 & 0 & 1 & 0 & 1 & 1 \\
\hline
\end{tabular}

Tabla 4. Equipos eléctricos en las tres zonas de Bolivia

Fuente: Elaboración propia, 2021.

\subsection{Optimizaciones y regresión multivariable}

Para realizar las optimizaciones se considera las siguientes tecnologías para sistemas aislados:

generadores a diésel y sistemas fotovoltaicos (PV), que acumulan energía en baterías; para sistemas concentrados la opción tecnológica es la red eléctrica; los parámetros tecno-económicos de las soluciones que se platean se muestran en las Tablas 5 y 6. 
JOURNAL BOLIVIANO DE CIENCIAS - Vol. 17 - Número 50

ISSN: $2075-8944$

Universidad Privada del Valle - Bolivia

https://doi.org/10.52428/20758944.v17i50.2

\begin{tabular}{|c|c|c|c|}
\hline \multicolumn{2}{|c|}{ Información tecno-económicos sistemas aislados } \\
\hline Parámetro & Unidades & Sistemas híbridos & Sistema para hogares \\
\hline Precio del Diesel & US\$/litro & 0.53 & 700 \\
\hline $\begin{array}{c}\text { Costo de inversión de las } \\
\text { baterías }\end{array}$ & US\$/kWh & 1250 & 1500 \\
\hline $\begin{array}{c}\text { Costo de inversión de los } \\
\text { PV }\end{array}$ & US\$/kW & 1480 & 1480 \\
\hline $\begin{array}{c}\text { Costo de inversión de los } \\
\text { generadores }\end{array}$ & US\$/kW & & 1 \\
\hline $\begin{array}{c}\text { Probabilidad de perdida } \\
\text { de carga }\end{array}$ & & 0 & \\
\hline
\end{tabular}

Tabla 5. Parámetros tecno-económicos para los sistemas aislados

Fuente: Elaboración propia, 2021.

\begin{tabular}{|c|c|c|}
\hline \multicolumn{3}{|c|}{ Información tecno-económica sistema interconectado } \\
\hline Parámetro & Unidades & Valores \\
\hline Perdidas de la red & $\%$ & 18,3 \\
\hline Distancia de la red de Medio voltaje & $\mathrm{km}$ & 50 \\
\hline Costo de línea alto voltaje $(115 \mathrm{kV})$ & $\mathrm{US} \$ / \mathrm{km}$ & 117000 \\
\hline Costo de línea alto voltaje $(69 \mathrm{kV})$ & $\mathrm{US} \$ / \mathrm{km}$ & 99000 \\
\hline Costo de línea medio voltaje $(33 \mathrm{kV})$ & $\mathrm{US} \$ / \mathrm{km}$ & 9000 \\
\hline Costo de línea de bajo voltaje $(0.24 \mathrm{kV})$ & US\$/km & 5000 \\
\hline Transformadores $(50 \mathrm{kVA})$ & US\$ & 3500 \\
\hline Max nodos por transformador & nodos & 300 \\
\hline Subestación (400 kVA) & US\$ & 10000 \\
\hline Subestación (1000 kVA) & US\$ & 25000 \\
\hline Costo de conectarse a la red & US\$/hogares & 150 \\
\hline
\end{tabular}

Tabla 6. Parámetros tecno-económicos para la red interconectada

Fuente: Elaboración propia, 2021. 
En la Tabla 7 se muestran los resultados promedio de los procesos de dimensionamiento. Debido a la mayor radiación existente en las zonas del altiplano, existen mayor capacidad instalada de baterías y PV que en las zonas bajas. Los sistemas para hogares basados exclusivamente en PV son considerablemente más caros que una microrred, debido a que, pierden la confiabilidad que los generadores dan al sistema, por lo que tienen que instalar una capacidad instalada de PV y baterías, alta en relación con la demanda pico del sistema. $\mathrm{El} \mathrm{R}^{2}$ de las regresiones es bastante alto, llegando a superar 0,97 en todos los casos.

Para los sistemas PV para hogares dispersos se usó un modelo estándar de 50 - 100 W por unidad, con un costo aproximado de 1000 dólares que incluye los servicios de distribución y verificación de funcionamiento, bajo una lógica de uso de sistemas fotovoltaicos de tercera generación (ErasAlmeida, 2019), de manera de poder abastecer la mínima energía requerida por los habitantes de un hogar en las zonas rurales de Bolivia.

En otra fase o, si el usuario así lo requiera, se podría integrar nuevos equipos ya sea separados del sistema principal o añadiendo más capacidad. Esto se hace de esta manera porque abastecer toda la demanda en un sistema distribuido puede elevar el costo a más de 5000 dólares por unidad.

\begin{tabular}{|c|c|c|c|}
\hline Equipo & $\begin{array}{c}\text { Microrred } \\
\text { híbrida tierras } \\
\text { altas }\end{array}$ & $\begin{array}{c}\text { Microrred } \\
\text { híbrida } \\
\text { amazonias }\end{array}$ & $\begin{array}{c}\text { Microrred } \\
\text { híbrida } \\
\text { chaco }\end{array}$ \\
\hline $\begin{array}{c}\text { Costo promedio de la } \\
\text { energía (USD/kWh) }\end{array}$ & 0,25 & 0,27 & 0,27 \\
\hline $\begin{array}{c}\text { Capacidad instalada del } \\
\text { generador de diésel (kW) }\end{array}$ & 60 & 18 & 36 \\
\hline $\begin{array}{c}\text { Capacidad instalada del PV } \\
(\mathrm{kW})\end{array}$ & 212 & 132 & 132 \\
\hline $\begin{array}{c}\text { Capacidad instalada de las } \\
\text { baterías (kWh) }\end{array}$ & 476 & 206 & 205 \\
\hline $\begin{array}{c}\text { Penetración de energía } \\
\text { renovable (\%) }\end{array}$ & 63 & 59 & 59 \\
\hline
\end{tabular}

Tabla 7. Valores promedios de los resultados de las optimizaciones

Fuente: Elaboración propia, 2021. 
JOURNAL BOLIVIANO DE CIENCIAS - Vol. 17 - Número 50

ISSN: 2075-8944

Universidad Privada del Valle - Bolivia

https://doi.org/10.52428/20758944.v17i50.2

\subsection{Resultados de la estimación de costos de electrificación}

Se dejó correr el algoritmo de OnSSET hasta alcanzar un 92\% de la población boliviana. A partir de ahí, se cerró la brecha con sistemas aislados híbridos para las poblaciones entre 40 y 560 hogares. Debido a la gran probabilidad de que las comunidades con menos de 40 hogares se encuentren dispersas, se decidió dotarlas con sistemas individuales.

El costo de inversión necesario para poder electrificar a Bolivia al 100\% se divide en 2 (Tabla 8).

\begin{tabular}{|l|c|c|c|c|}
\hline \multicolumn{1}{|c|}{ Tecnología } & \multicolumn{4}{|c|}{ Resultados de electrificación } \\
\hline & $\begin{array}{c}\text { Población } \\
\text { electrificada 2012- } \\
\mathbf{2 0 2 5}\end{array}$ & $\begin{array}{c}\text { Costos de } \\
\text { inversión, } \\
\text { millones (US\$) }\end{array}$ & $\begin{array}{c}\text { Nueva capacidad } \\
\text { (MW) }\end{array}$ & $\begin{array}{c}\text { Costo capital } \\
\text { promedio } \\
\text { (US\$/hogar) }\end{array}$ \\
\hline Extensión de red & 3149932 & 1027 & 262 & 1174 \\
\hline Mini red híbrida & 565074 & 479 & 72 & 2898 \\
\hline Hogar PV & 368677 & 108 & 11 & 1000 \\
\hline \multicolumn{1}{|c|}{ Total } & $\mathbf{4 0 8 3 6 8 3}$ & $\mathbf{1 6 1 4}$ & $\mathbf{3 4 5}$ & -- \\
\hline
\end{tabular}

Tabla 8. Valores promedios de los resultados de las optimizaciones

Fuente: Elaboración propia, 2021.

Son alrededor de 1027 millones de US\$ para la extensión de la red, cantidad que posiblemente ya ha sido invertida, ya que los últimos informes apuntan a un rango entre $88 \%$ a $92 \%$ de cobertura por la red. Sin una nueva planificación de líneas de alta tensión, es muy difícil que esta tecnología logre cubrir una cantidad significativa de personas en un futuro próximo. Debido a la dificultad de hacer seguimiento a la multitud de proyectos realizados en todo el país, es difícil calcular una cantidad exacta de inversión realizada hasta la fecha. La segunda parte de estos costos son las inversiones necesarias para llegar a las poblaciones aisladas, el monto necesario es de 587 millones de dólares. 


\section{JOURNAL BOLIVIANO DE CIENCIAS - Vol. 17 - Número 50}

ISSN: $2075-8944$

Universidad Privada del Valle - Bolivia

https://doi.org/10.52428/20758944.v17i50.2

\section{DISCUSIÓN Y CONCLUSIONES}

Aunque el costo total calculado es elevado, el valor está en línea con lo declarado por el Viceministerio de Electricidad y Energías Alternativas, de alrededor 1976 millones de USD (Ministerio de Hidrocarburos y Energía, 2014). Las cantidades son próximas, pero es difícil comparar ambos costos sin tener el detalle de las hipótesis asumidas por las personas que realizaron los cálculos. Aun así, podemos concluir que ambos están en el rango de valores esperados para este tipo de tecnologías.

Lograr 100\% de electrificación significaría una inversión importante de recursos económicos y humanos para el Estado boliviano. Una parte significativa ya ha sido invertida, llegando a casi un 90\% de la población. A pesar de esto, existen más de 8000 comunidades que no han sido alcanzadas por la red que representan 273286 familias.

Hoy en día, la inversión de alrededor 587 millones de dólares para dar electricidad a estas comunidades es una cantidad accesible para un país como Bolivia. De este universo sin acceso a la electricidad, la población más vulnerable son 108000 familias rurales aisladas, las que sin duda tienen como la mejor opción técnico-económica a los sistemas fotovoltaicos individuales.

La barrera más significativa es la capacidad de Bolivia para llevar a cabo la cantidad de proyectos necesarias para cerrar la brecha. Es importante continuar con la formación de personal técnico y administrativo que sea capaz de diseñar y ejecutar este tipo de proyectos con suma urgencia. Así mismo, es necesario conformar alianzas estratégicas entre diferentes organizaciones y fortalecer el marco institucional, considerando que la cuestión energética para la población aislada constituye un desafío técnico-económico y de gestión, radicalmente diferente de los sistemas convencionales, en términos de densidad, estructura y prácticas de regulación y gestión.

Un actor importante que se debe incorporar ya en los procesos de planificación de detalle de las inversiones de implementación, son los Municipios, pues en este nivel se tiene el contacto directo con los potenciales beneficiarios, y son ellos los que conocen de primera mano las comunidades y familias afectadas con la ausencia del servicio de electricidad. 
JOURNAL BOLIVIANO DE CIENCIAS - Vol. 17 - Número 50

ISSN: $2075-8944$

Universidad Privada del Valle - Bolivia

https://doi.org/10.52428/20758944.v17i50.2

\section{REFERENCIAS}

Arraiz, I., Calero, C. (2015). From Candles to Light: The Impact of Rural Electrification. InterAmerican Development Bank.

Autoridad de Fiscalización y control Social de Electricidad. (2015). Memoria estadística 2015. La Paz. Bolivia

Balderrama, S., Lombardi, F., Stevanato, N., Peña, G., Colombo, E., Howells, M., Quoilin, S. (2020). Surrogate models for energy planning: Application to Bolivian lowlands isolated communities. Energy, On Press

Banco Mundial. (1 de junio de 2020). Datos de libre acceso del Banco Mundial. Obtenido de $\begin{array}{lllll}\text { Acceso a la } & \% \text { electricidad de población): }\end{array}$ https://datos.bancomundial.org/indicador/EG.ELC.ACCS.ZS?locations=ZJ

Catálogo GeoBolivia - GeoBolivia. (n.d.). Retrieved October 9, 2019, from https://geo.gob.bo/geonetwork/srv/spa/catalog.search\#/metadata/3f73d133-031c-4ed2a7c3-e4569f03c5a6

CEPAL. (Noviembre de 2017). Avances en Materia de Energías Sostenibles en America Latina y el Caribe. Resultados del Marco de Seguimiento Mundial, informe 2017. Unidad de Recursos Naturales y Energía. Santiago de Chile: Naciones Unidas.

Cobertura del Servicio Eléctrico a Nivel Comunidades Censo 2012 - Explora Mapas example.com. (n.d.). Retrieved October 9, 2019, from http://sigvmeea.minenergias.gob.bo/maps/73

Eras-Almeida, A.; Fernandez, M., Eisman, J., Martin, J. G., Caamaño, E., Egido-Aguilera, M. (2019). Lessons learned from rural electrification experiences with third generation solar home systems in latin America: Case studies in Peru, Mexico, and Bolivia. Sustainability, 11(24), 7139. https://doi.org/10.3390/su11247139

Feres, J. C., Mancero, X. (n.d.). El método de las necesidades básicas insatisfechas (NBI) y sus aplicaciones en América Latina.

Fernandez, M. (2010). Rol e impacto socioeconómico de las energías renovables en Bolivia. La Paz, Bolivia: CEDLA.

Instituto Nacional de Estadística. (2019). Estadísticas Demográficas de Bolivia.

Jimenez, R. (2017). Development Effects of Rural Electrification. Inter American Development Bank, Infrastructure and Energy Sector. Inter American Development Bank. 
JOURNAL BOLIVIANO DE CIENCIAS - Vol. 17 - Número 50

ISSN: $2075-8944$

Universidad Privada del Valle - Bolivia

https://doi.org/10.52428/20758944.v17i50.2

Lombardi, F., Balderrama, S., Quoilin, S., Colombo, E. (2019). Generating high-resolution multienergy load profiles for remote areas with an open-source stochastic model. Energy, 117, 433-444. https://doi.org/10.1016/j.energy.2019.04.097

Mandelli, S., Merlo, M., Colombo, E. (2016). Novel procedure to formulate load profiles for offgrid rural areas. Energy for Sustainable Development, 31, 130-142. https://doi.org/10.1016/j.esd.2016.01.005

Mentis, D., Howells, M., Rogner, H., Korkovelos, A., Arderne, C., Zepeda, E., Scholtz, E. (2017). Lighting the World: the first application of an open source, spatial electrification tool (OnSSET) on Sub-Saharan Africa. Environmental Research Letters, 12(8), 085003. https://doi.org/10.1088/1748-9326/aa7b29

Ministerio de Hidrocarburos y Energía. (2014). Plan Eléctrico del Estado Plurinacional de Bolivia 2015- 2025. Ministerio de Hidrocarburos y Energía, La Paz, Bolivia.

Peña. G., Balderrama, S., Lombardi. F., Stevanato. N., Sahlberg, A., Howells. M., Colombo, E., Quoilin. S. (2020). Incorporating high-resolution demand and techno-economic optimization to evaluate micro-grids into the Open Source Spatial Electrification Tool. Energy for Sustainable Development, 56, 98-118. https://doi.org/10.1016/j.esd.2020.02.009

Scmukler, M., Garrido, S. (2016). Electrificación Rural en Argentina. Adecuación Socio-técnica del Programa PERMER en la Provincia de Jujuy. XXXIX Reunión de Trabajo de la Asociación Argentina de Energías Renovables y Medio Ambiente (ASADES), Buenos Aires, Argentina: ASADES.

UNDP. (2018). www.undp.org.Obtenido de Objetivos de Desarrollo Sostenible: http://www.undp.org/content/undp/es/home/sustainable-development-goals.html

Vieira de Carvalho, A. (25 de febrero de 2015). Planificación para el Acceso Universal a la Energía (Spanish-Language Webinar). Recuperado de Acceso a la Energía Electrica. Situacion en Latinoamerica y el Caribe. Recuperdo de: https://cleanenergysolutions.org/sites/default/files/documents/planificacion-para-accesouniversal-a-electricidad-arnaldo-bid.pd

Fuentes de financiamiento: Esta investigación ha sido auspiciada por la Red de Soluciones para el Desarrollo Sostenible - SDSN - Bolivia en su convocatoria de Estudios Transversales 2020, por lo que los autores expresan su agradecimiento al apoyo de esta iniciativa.

Declaración de conflicto de intereses: Los autores declaran que no tienen ningún conflicto de interés. 


\section{JOURNAL BOLIVIANO DE CIENCIAS - Vol. 17 - Número 50}

ISSN: $2075-8944$

Universidad Privada del Valle - Bolivia

https://doi.org/10.52428/20758944.v17i50.2

Copyright (c) 2021 Miguel Fernández Fuentes; Evelyn Cardozo R.; Jaime Zambrana Vargas; Gabriela Peña; Sergio Balderrama; Claudia Sánchez; Alejandro Soto; Sylvain Quoilin

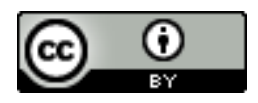

Este texto está protegido por una licencia $\underline{\text { Creative Commons }} \underline{4.0}$.

Usted es libre para Compartir — copiar y redistribuir el material en cualquier medio o formato - y Adaptar el documento remezclar, transformar y crear a partir del material - para cualquier propósito, incluso para fines comerciales, siempre que cumpla la condición de:

Atribución: Usted debe dar crédito a la obra original de manera adecuada, proporcionar un enlace a la licencia, e indicar si se han realizado cambios. Puede hacerlo en cualquier forma razonable, pero no de forma tal que sugiera que tiene el apoyo del licenciante o lo recibe por el uso que hace de la obra.

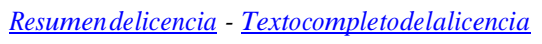

